\title{
The Beijing-Tianjin-Hebei Region's Analysis of Air Quality Assessment
}

\author{
Xiaohui Sun ${ }^{1, a}$ \\ ${ }^{1}$ School of Environmental Science and Engineering Department, North China Electric Power \\ University, Baoding 071003, China \\ asunxiaohui77@126.com
}

Keywords: Source analysis; Air quality; PCA; Kriging; Interaction effects

\begin{abstract}
Control air pollution, improve the ecological environment is an important breakthrough of the coordinated development of beijing-tianjin-hebei. So this paper analyzed the air quality of Beijing-Tianjin-hebei, give their comprehensive evaluation analysis. Analysis the pollutant source and get the interaction relations between different regions from analyzing meteorological data. In the process of air quality comprehensive evaluation, using the principal component analysis to handle the six air index, carry on the solution of the characteristic roots and characteristic vectors, to get the comprehensive expression of each component. From the comprehensive expression, we calculate the comprehensive evaluation value of each city and sorting. Using the kriging interpolation method, map out air quality comprehensive evaluation. Using Pearson product to analyze correlation coefficient's influence on the regional air quality between cities, get the interaction relations between different regions. Aiming to guide the practice of environmental protection measures, help to control the atmospheric pollution.
\end{abstract}

\section{Introduction}

Beijing-Tianjin-Hubei region as China's political and cultural center is the national management, foreign exchange, and technology trade and transport hub. Promoting coordinated development of the beijing-tianjin-hebei region is the major strategic decision that the party central committee and the state council make. And air pollution becomes the major bottlenecks that restrict the development of the region.

We should put control air pollution and improve the ecological environment as an important breakthrough of the coordinated development of beijing-tianjin-hebei. So the analysis of spatial and temporal distribution of air pollution in the beijing-tianjin-hebei region, the interaction relationship between the regions for atmospheric pollution control has important significance. It can help to control the air pollution and promote the coordinated development of the regions.

This paper presents a comprehensive evaluation method of air quality and adopts the national air quality data to analyze. Also analysis of range air quality around the interactional relationship, and the regional pollution source.

\section{A Comprehensive Evaluation Method of Air Quality}

Model of Principal Components Analysis. For quantitative analysis of air pollution, it is necessary to building the appraisal model of air quality. At present our country adopts the Air Quality Index (Air Quality Index, AQI) to evaluate the Air Quality.[1] But its drawback is that part of the data in the process of comprehensive inevitably will be lost, and can't reflect the pollution levels of pollutants in the air and the relationship between them, therefore use the principal components analysis to the comprehensive evaluation on the various pollutants in the air.

Based on May 13, 2014 to March 11, 2015 national air quality historical data [2], Sort out Beijing, Tianjin, Shijiazhuang, Tangshan, Qinhuangdao, Handan, Baoding, Zhangjiakou, Chengde, Langfang, Cangzhou, Hengshui, Xingtai thirteen cities, 9,12,3 six months 122 days each pollutant (PM2.5, $\mathrm{PM} 10, \mathrm{SO}_{2}, \mathrm{NO}_{2}, \mathrm{O} 3$ and $\mathrm{CO}$ ) values. Choose a day 0 points measured 24-hour average data to 
represent the air quality in a day. [3] For six-air quality evaluation index X1, X2... X6 (PM2.5, PM10, $\mathrm{SO}_{2}, \mathrm{NO}_{2}, \mathrm{O} 3$ and $\mathrm{CO}$ ) to use principal component analysis.

In order to facilitate the comparison of the degree of air quality between different quarters, first put 122 days of 13 cities in six pollutants value unified standardizing, then respectively the principal component analysis for four months. The characteristic vector of the characteristic root is shown in the Table 1 [4]:

Table 1 The characteristic vector of the characteristic root

\begin{tabular}{c|c|c|c|c|c|c}
\hline Feature Vector & $\mathrm{x} 1$ & $\mathrm{x} 2$ & $\mathrm{x} 3$ & $\mathrm{x} 4$ & $\mathrm{x} 5$ & $\mathrm{x} 6$ \\
\hline First & 0.4781 & 0.3867 & 0.4063 & 0.4735 & 0.0188 & 0.4819 \\
\hline Second & 0.0805 & 0.4299 & -0.0679 & -0.1947 & 0.8499 & -0.2094 \\
\hline Third & -0.3918 & -0.4973 & 0.6442 & 0.0912 & 0.3954 & 0.1395 \\
\hline Fourth & 0.0335 & -0.3931 & -0.6288 & 0.4742 & 0.3362 & 0.3332 \\
\hline Fifth & -0.4288 & 0.2905 & 0.016 & 0.6917 & -0.0694 & -0.4982 \\
\hline Sixth & 0.653 & -0.4235 & 0.1402 & 0.1619 & 0.0559 & -0.5875 \\
\hline
\end{tabular}

The expression of the 6 principal components is obtained.

Put the standardized data into the expression, and then get each principal component values for each city. With its principal component contribution rate as weights, constructs the comprehensive evaluation model of principal component, is

$$
Z=0.557 y_{1}+0.195 y_{2}+0.120 y_{3}+0.059 y_{4}+0.040 y_{5}+0.029 y_{6}
$$

Put feature vector into (1), can get

$$
Z=0.238 \tilde{x}_{1}+0.216 \tilde{x}_{2}+0.258 \tilde{x}_{3}+0.297 \tilde{x}_{4}+0.242 \tilde{x}_{5}+0.227 \tilde{x}_{6}
$$

This is the Model of Principal Components Analysis.

Historical Data Analysis. In march the city's average value of six pollution generations into the standardized data type, the higher the value, the lower the air quality. We can get the Available cities' spring air quality value and the result of sort.

Table 2 The result of sort

\begin{tabular}{l|c|c|c|c|c|c|c}
\hline City & Zhangjiakou & Chengde & Changzhou & Handan & Beijing & Tianjin & Langfang \\
\cline { 1 - 7 } Ranking & 1 & 2 & 3 & 4 & 5 & 5 & 7 \\
\hline Value & -0.62 & -0.43 & -0.08 & -0.07 & -0.06 & -0.06 & -0.05 \\
\hline \hline City & Qinhuangdao & Hengshui & Shijiazhuang & Xingtai & Tangshan & Baoding & \\
\cline { 1 - 6 } Ranking & 8 & 9 & 10 & 11 & 12 & 13 & \\
\hline Value & 0.08 & 0.13 & 0.35 & 0.61 & 0.7 & 0.72 & \\
\hline
\end{tabular}

The figure shows that the air quality of the best city of is Zhangjiakou. chengde, and cangzhou is better also. baoding, tangshan's air quality is the worst.

The Relationship between Air Pollutants and Season. By the same time, we established the summer, autumn and winter air quality comprehensive evaluation model, according to the pollution coefficient of variation, and we can analyze the content in the air pollutants and season relationship: 


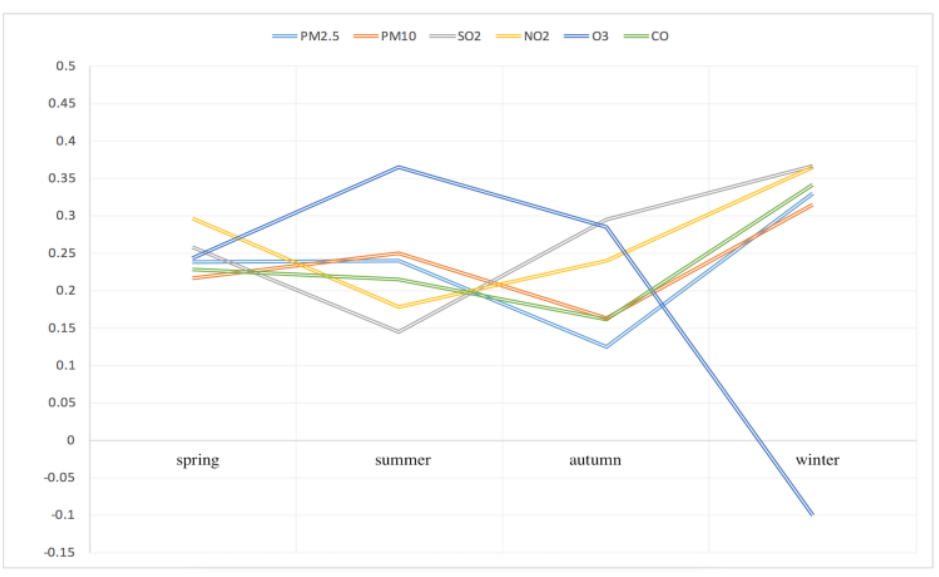

Figure 1. The air pollutants and season relationship

The figure shows that in addition to the ozone other contaminants by month is first fall again rise, especially in the tendency of the sulfur dioxide is most obvious, this kind of circumstance is in line with the seasonal climate. heating season is late autumn to early winter season, a lot of coal combustion is bound to cause the increase of sulfur dioxide in the air.

Figure of sulfur dioxide and nitrogen dioxide values, this is because both have similar pollution sources. Pollution is mainly generated from combustion of the fuel, car tail gas, and the use of nitric acid plant tail gas. ${ }^{[5]}$ The summer precipitation in beijing-tianjin-hebei region is larger, humidity is bigger. These are the cause of these pollutants content decreased. Pm2.5 and pm10 trends basic same, this is due to both measure of particulate matter in the air has a certain relevance.

\section{Air Quality in Time and Space Distribution Characteristics}

Kriging. Use Surfer software, according to the longitude and latitude of the city of beijing-tianjin-hebei region 13 city build property sheet, enter the season average of each city air quality, the integrated evaluation on the kriging interpolation method [6]is used to for the optimal interpolation, drawing the air quality comprehensive evaluation's contour map.
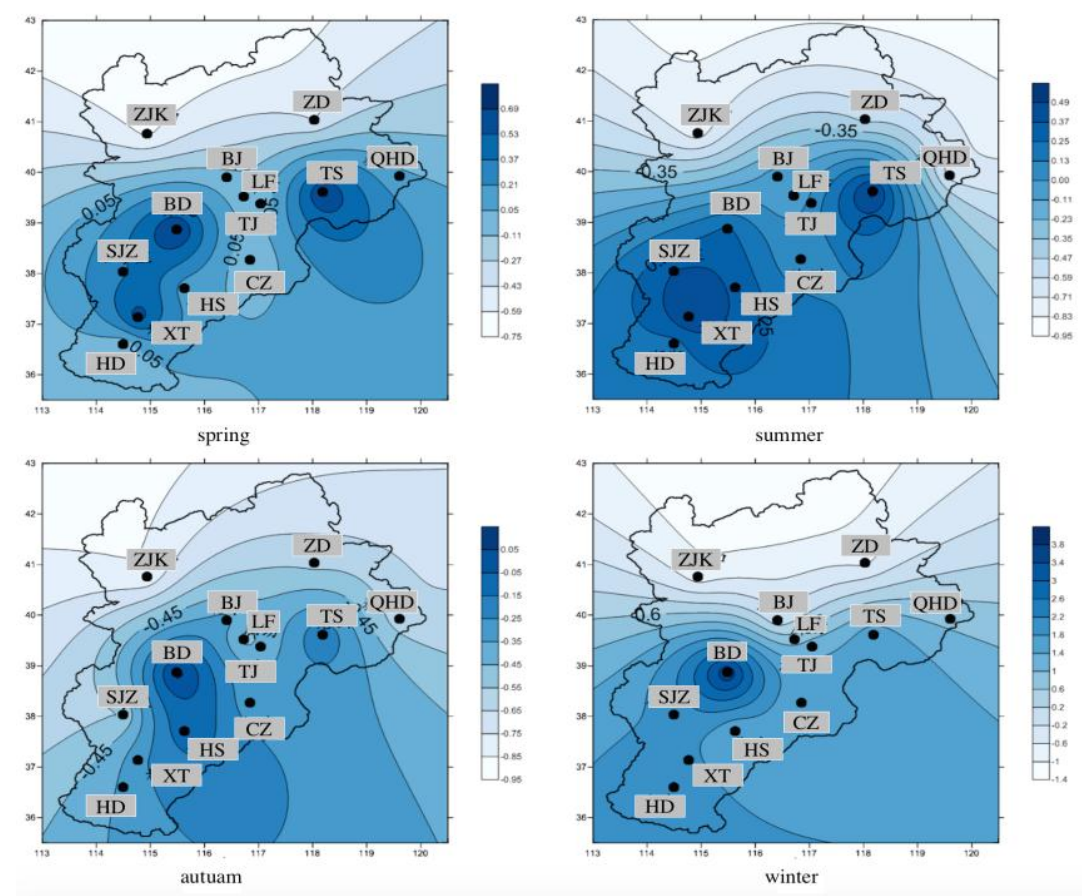

Figure 2. The air quality comprehensive evaluation's contour map 
The figure shows that the air quality of spring air distribution for the integrated evaluation: the northern region is low, from north to south gradually rise, largely in zonal distribution; Southern air quality overall on the high side, the integrated evaluation in tangshan, baoding, xingtai is high value center of diminishing.

\section{The Interaction Relations between Different Regions}

With the development of urbanization, there has no obvious boundary between the two cities. And because of the airflow, all kinds of air pollutant under the influence of meteorological conditions interaction, mutual influence. ${ }^{[7]}$ In order to measure the air qualities relationship between cities, we introduced the Pearson product moment correlation coefficient, by quarter time standard, use the last work out of 13 cities $^{[8]}$ in the beijing-tianjin-hebei region four quarters, the air quality evaluation based on Pearson product moment correlation coefficient influence on regional air quality between cities is analyzed. ${ }^{[9]}$

In spring, for example, the result as follows:

Table 3 Comprehensive evaluation value of correlation coefficient analysis

\begin{tabular}{cccccccccccccc}
\hline City & TJ & SJZ & TS & QHD & HD & BD & ZJK & CD & LF & CZ & HS & XT & BJ \\
\hline TJ & 1 & & & & & & & & & & & \\
SJZ & 0.56 & 1 & & & & & & & & & & \\
TS & 0.86 & 0.22 & 1 & & & & & & & & & & \\
QHD & 0.83 & 0.34 & 0.93 & 1 & & & & & & & & & \\
HD & 0.56 & 0.58 & 0.18 & 0.34 & 1 & & & & & & & & \\
BD & 0.69 & 0.75 & 0.35 & 0.51 & 0.95 & 1 & & & & & & \\
ZJK & -0.71 & -0.22 & -0.75 & -0.50 & 0.02 & -0.14 & 1 & & & & & \\
CD & -0.52 & -0.30 & -0.63 & -0.54 & -0.12 & -0.33 & 0.73 & 1 & & & & \\
LF & 0.87 & 0.51 & 0.57 & 0.47 & 0.68 & 0.71 & -0.66 & -0.39 & 1 & & & \\
CZ & 0.33 & 0.78 & -0.08 & 0.08 & 0.53 & 0.56 & 0.17 & 0.34 & 0.35 & 1 & & & \\
HS & 0.11 & 0.40 & -0.36 & -0.25 & 0.63 & 0.47 & 0.36 & 0.59 & 0.38 & 0.79 & 1 & & \\
XT & 0.81 & 0.88 & 0.63 & 0.74 & 0.56 & 0.78 & -0.45 & -0.57 & 0.61 & 0.53 & 0.09 & 1 & \\
BJ & 0.68 & 0.17 & 0.70 & 0.45 & 0.10 & 0.23 & -0.97 & -0.78 & 0.71 & -0.26 & -0.34 & 0.41 & 1 \\
\hline
\end{tabular}

In Beijing, for example, can be seen from the table, the air quality of Beijing and langfang correlation coefficient is 0.71 , the integrated evaluation has strong correlation. High correlation coefficient between these cities shows that the pollution in the same area, these cities has similar characteristics of air pollution. In other words, when one of these cities urban heavy air pollution phenomenons occurs, one or several of the other cities also can appear the corresponding pollution.

The above conclusion can guide related policy specified, let people more clearly aware of their state. Such as the air quality had a greater influence on the correlation between different cities. Pollution of urban agglomeration with similar characteristics, presents the obvious regional characteristics. To comprehensive control of beijing-tianjin-hebei regional air quality, the need for air pollution control in the process of joint prevention and control for the whole area, rather than to a single region governance. [10]

\section{References}

[1] Yele Sun,Zifa Wang,Pingqing Fu,Qi Jiang,Ting Yang,Jie Li,Xinlei Ge. The impact of relative humidity on aerosol composition and evolution processes during wintertime in Beijing, China[J]. Atmospheric Environment . 2013 
[2] Information on http://beijinggair.sinaapp.com/, 2015/5/2

[3] Stein D C,Swap Rt J,Greco Steven,et al.Haze layer characterization and associated meteorological controls along the eastern coastal region of southern Africa. Journal of Geophysical Research . 2003

[4] Shuanghong Qu. Several common improvement method based on principal component analysis [J]. Statistics and decision. 2011(05)

[5] Pan Y. P.,Wang Y. S.,Tang G. Q.,Wu D.. Spatial distribution and temporal variations of atmospheric sulfur deposition in Northern China: insights into the potential acidification risks [J]. Atmospheric Chemistry and Physics . 2013 (295)

[6]
A Basic
Understanding
of Surfer
Gridding
Methods, http://www.goldensoftware.com/newsletter/issue71-surfer-gridding-methods-part\#, 2015/5/

[7] Peter C. B. Phillips.New tools for understanding spurious regressions. Econometrica . 1998

[8] Yuesi Wang, Xiyan Ren, Dongsheng Ji, Junqang Zhang, Jie Sun, Fankun Wu. Characterization of volatile organic compounds in the urban area of Beijing from 2000 to 2007 [J]. Journal of Environmental Sciences . 2012 (1)

[9] Zang Z,Salermo J J,Yu P S.Applying data mining in investigating money laundering crimes. SIGKDD . 2003

[10] Jinnan Wang. Regional air pollution zone spreading the theories and methods of analysis [J]. Environment and sustainable development. 2012(05). 